\title{
VIEWPOINT
}

\section{Time to take epigenetic inheritance seriously}

\author{
Marcus E Pembrey ${ }^{*} 1$ \\ ${ }^{1}$ Clinical and Molecular Genetics Unit, Institute of Child Health, University College London, London, UK
}

European Journal of Human Genetics (2002) 10, 669-671. doi:10.1038/sj.ejhg.5200901

Keywords: epigenetic inheritance; ancestors' nutrition; diabetes; imprinting; BORIS

\section{Rich pickings from the past}

Any study of transgenerational effects needs information from across the generations, and this is not easy to obtain in humans. There is little research on the effect of exposures in grandparents on outcomes in their grandchildren, but one such study, ${ }^{1}$ reported on pages $682-688$ of this issue, obliges us to confront the possibility of epigenetic inheritance down the male line. Herein lies the importance for human geneticists of the work of Kaati, Bygren and Edvinsson from Umea University, Sweden. Building on their interest in early nutritional influences on cardiovascular mortality, they have exploited records of annual harvests from an isolated community in northern Sweden that go back as far as 1799 to explore the effects of food availability across three generations.

Earlier work $^{2}$ by the team on a cohort born in 1905 showed a remarkable effect of food availability during the slow growth period (SGP) just before puberty of the paternal grandfather on the longevity of the probands. Scarcity of food in grandfather's SGP was associated with a significantly extended survival of his grandchildren for many years, whilst food abundance was associated with a greatly shortened life span of the grandchildren. There are only four possible explanations; chromosomal transmission of nutritionally-induced epigenetic modifications, intense genetic selection through differential survival /fertility, a statistical quirk or hidden bias producing a false association, or some mechanism of inheritance yet to be discovered. In the present study ${ }^{1}$ Kaati and colleagues have enlarged the sample with two new cohorts born in 1890 and 1920 in

\footnotetext{
*Correspondence: Professor Marcus Pembrey, Picketts South, Picketts Lane, Redhill, Surrey RH1 5RG, UK. Tel/fax: +44 (0)1293 771202; E-mail: mpembrey@picketts.u-net.com

Received 26 July 2002; revised 6 August 2002; accepted 29 August 2002
}

order to have the power to look specifically at cardiovascular and diabetes related deaths. The latter outcome was chosen because imprinted genes have been implicated in diabetes risk.

The first thing to say is that the shorter survival of probands when the paternal grandfather had been exposed to plenty of food during his SGP was replicated in the newly studied 1890 cohort, although this association could not be demonstrated in the 1920 cohort. Overall they show that cardiovascular mortality was reduced with poor availability of food in the father's SGP, but also with good availability in the mother's SGP. This reciprocal effect of parental nutrition is intriguing in itself, but the most striking result comes with diabetes. If the paternal grandfather was exposed to a surfeit of food during his SGP, then the proband had a fourfold excess mortality related to diabetes (OR 4.1, 95\% c.i.1.33-12.93, $P=0.01$ ) when age at death and the effects of possible over eating among parents and grandparents during their respective SGP were taken into account. Interestingly a father's exposure to a surfeit of food during his SGP tended to protect the proband from diabetes (OR 0.13, 95\% c.i. $0.02-1.07, P=0.06$ ), hinting at some 'see-saw' effect down the generations. The more unexpected the result, the more important it is to replicate the findings on other cohorts. This should be possible in northern Sweden thanks, in part, to the regional harvest records that were demanded by His Majesty the King in times gone by.

\section{What could be going on?}

These are, potentially, very important findings hinting at some, as yet undiscovered, transgenerational mechanism that 'captures' nutritional information from the previous generation(s). Could there be a more mundane explanation? At a time of relatively high infant mortality and 
with the possibility of swings in food supply influencing fertility, genetic selection has to be considered, although it would have to be very intense to produce this effect over three generations. The authors addressed this question focusing on longevity, food availability in infancy and sibship size. They found no evidence of intense selection, but did come up with one curious finding. The number of children in the probands' sibship was lower when the paternal grandfather faced a surfeit of food during the SGP, but higher when the father, likewise, faced a good food supply during his SGP. It is difficult to see how this bias could generate the main results. However, at risk of over-interpreting the data, it is interesting to note that the same combination of exposures down the male line influences both sibship size in the third generation and the diabetic risk in these grandchildren. Could the same transgenerational (adverse) influence cause both subfertility and/or increased embryonic/foetal loss and a diabetic susceptibility in those offspring who survive? In this case the transgenerational response to swings in nutrition could have evolved primarily in relation to the regulation of reproduction, with the diabetic risk being a secondary consequence.

\section{The plausibility of epigenetic inheritance}

One of the tests of the causal nature of an association is biological plausibility. There has been a distinct reluctance to take the possibility of human epigenetic inheritance seriously, despite experimental evidence in mammals. ${ }^{3-7}$ Even, the recent 'rheostat model' for a rapid and reversible form of imprinting-dependent evolution, ${ }^{8}$ whilst acknowledging the potential for non-Mendelian or transgenerational effects, stops short of proposing any mechanism.

Part of the reluctance to embrace the idea of epigenetic inheritance may be a mistrust of any hypothesis with a Lamarkian flavour, but the main reason has been the lack of compelling human observations and a plausible molecular mechanism that could be investigated experimentally. When I first suggested ${ }^{9}$ that imprinted genes were good candidates to mediate nutrition-linked transgenerational effects on growth, little was known about imprint establishment or erasure and there were no published human data that assessed nutritional influences down the male line. Transmission down the female line is always open to more than one interpretation, since one generation resides inside another permitting a cascade of trans-placental metabolic effects down the generations.

\section{A possible model}

Kaati and colleagues ${ }^{1}$ rightly point out that if imprinted genes are involved, the INS-IGF2-H19 imprinted domain is a good candidate. It is paternally imprinted (i.e the gametic imprints are established during spermatogenesis) ${ }^{10}$ and variation at the INS VNTR, at least, is associated with diabetes risk (both types). ${ }^{11,12}$ Pertinent are human data showing that the father's untransmitted INS VNTR allele can influence the effect of the transmitted allele on the child's diabetes risk (type 1 in this case), ${ }^{11}$ indicating that a transgenerational mechanism can operate on the INSIGF2-H19 imprinted domain. Even if we accept that some epigenotypes are transmitted and can adjust gene expression at this domain, the question remains; what does the adjusting and can it plausibly operate in a boy's testes during his SGP, say between 8 and 11 years?

Enter BORIS (Brother Of the Regulator of Imprinted Sites), a novel male germ-line-specific protein associated with epigenetic reprogramming events. ${ }^{13,14}$ BORIS shares the same DNA-binding domain as CTCF, the insulator protein involved in reading imprinting marks at the imprinting control region between IGF2 and H19, amongst many functions. ${ }^{15}$ CTCF is expressed ubiquitously in the soma and is absolutely necessary for cell survival, a role most likely performed by BORIS in the primary spermatocytes of the testis, where CTCF is silenced. Crucially, BORIS is up-regulated in primary spermatocytes to become silenced on activation of CTCF in post-meiotic germ-line cells. Furthermore, this BORIS to CTCF switch of expression takes place in association with erasure and re-establishment of methylation marks, respectively, as visualised by antibodies against $5 \mathrm{mC} .^{13}$ How directly this switch in gene expression is involved in imprint re-programming remains to be determined.

There is a paucity of information on the human prepubertal testis, but what studies ${ }^{16-18}$ there are indicate that prespermatocytes are present from 5 years. From about 8 years of age the proportion of boys (and seminiferous tubules) with primary spermatocytes increases until full spermatogenesis at puberty. Increasingly, some primary spermatocytes survive to progress through meiosis to spermatids. The SGP is therefore associated with the emergence of the first viable pools of spermatocytes and the beginning of re-programming of methylation imprints, just the kind of dynamic state in which a nutrition-sensing mechanism could operate. The nutritional state could directly effect imprint establishment (? by influencing the BORIS-CTCF switch) and/or trigger selective survival of those germ cells with a particular epigenetic state, assuming imprint re-programming is somewhat variable. Physiological metabolic and hormonal changes in response to nutritional stress could plausibly effect signalling pathways of modifying enzymes, which in turn could alter the posttranslational state of the CTCF/BORIS proteins leading to a change in their function. ${ }^{14}$

\section{Food for thought}

It seems that the Swedish studies have uncovered a nutrition-linked sperm-mediated transgenerational effect. Whilst one striking result relates to the grandfather's food availability, epigenetic transmission from just father to child would be sufficient to set up a cascade of metabolic 
responses down the generations. Independent replication is needed, but these observations should trigger entirely new lines of enquiry and at a time when we are getting an experimental handle on imprint re-programming.

\section{Acknowledgements}

I am grateful to Elena Klenova and Richard Sharpe for helpful discussions, whilst writing this commentary.

\section{References}

1 Kaati G, Bygren LD, Edvinsson S: Cardiovascular and diabetes mortality determined by nutrition during parents' and grandparents' slow growth period. Eur J Hum Genet 2002; 10: 682-688.

2 Bygren LO, Kaati G, Edvinsson S: Longevity determined by ancestors' overnutrition during their slow growth period. Acto Biotheoretica 2001; 49: 53-59.

3 Campbell JH, Perkins P: Transgenerational effects of drug and hormone treatments in mammals: a review of observations and ideas. Prog Brain Res 1988; 73: 535 -553.

4 Boucher BJ, Ewen SW, Stowers JM: Betel nut (Areca catechu) consumption and the induction of glucose intolerance in adult CD1 mice and in their F1 and F2 offspring. Diabetologia 1994; 37: 49-55.

5 Roemer I, Reik W, Dean W et al: Epigenetic inheritance of specific changes in gene expression in the mouse. Curr Biol 1997; 7: 277 280.

6 Morgan HD, Sutherland HG, Martin DI et al: Epigenetic inheritance at the agouti locus in the mouse. Nat Genet 1999; 23: $314-318$.

7 Wolff GL, Kodell RL, Moore SR et al: Maternal epigenetics and methyl supplements affect agouti gene expression in Avy/a mice. FASEB J 1998; 12: 949-957.
8 Beaudet AL, Jiang Y: A rheostat model for a rapid and reversible form of imprinting-dependent evolution. Am J Hum Genet 2002; 70: $1389-1397$.

9 Pembrey M: Imprinting and transgenerational modulation of gene expression; human growth as a model. Acta Genet Med Gemellol (Roma) 1996; 45: 111-125.

10 Judson H, Hayward BE, Sheridan E et al: A global disorder of imprinting in the human female germ line. Nature 2002; 416: $539-542$.

11 Bennett ST, Wilson AJ, Esposito L et al: Insulin VNTR allele-specific effect in type 1 diabetes depends on the identity of untransmitted paternal allele. Nat Genet 1997; 17: 350-352.

12 Ong KK, Phillips DI, Fall C et al: The insulin gene VNTR, type 2 diabetes and birth weight. Nat Genet 1999; 21: 262-263.

13 Loukinov DI, Pugacheva E, Vatolin S et al: BORIS, a novel male germ-line-specific protein associated with epigenetic reprogramming events, shares the same 11-zinc-finger domain with CTCF, the insulator protein involved in reading imprinting marks in the soma. PNAS 2002; 99: 6806-6811.

14 Klenova EM, Morse IIIHC, Ohlsson R, Lobanenkov VV: The novel $B O R I S+C T C F$ gene family is uniquely involved in the epigenetics of normal biology and cancer. Seminars in Cancer Biology 2002; 12: $399-414$.

15 Ohlsson R, Renkawitz R, Lobanenkov V: CTCF is a uniquely versatile transcription regulator linked to epigenetics and disease. Trends Genet 2001; 17: 520-527.

16 Muller J, Skakkebaek NE: Quantification of germ cells and seminiferous tubules by stereological examination of the testicles from 50 boys who suffered from sudden death. Int J Androl 1983; 6: $143-156$.

17 Nistal M, Paniagua R: Occurrence of primary spermatocytes in the infant and child testis. Andrologia 1984; 16: 532-536.

18 Trainer TD: Histology of the normal testis. Am J Surg Pathol 1987; 11: $797-809$. 